\title{
LORETA predicts electromagnetic sensitivity and "hearing voices" in a predictable, increasingly prevalent subpopulation: possible QEEG-based differential diagnosis
}

Michael A Persinger ${ }^{1,2,3,4^{*}}$, Linda S St-Pierre ${ }^{1,2}$ and Kevin S Saroka ${ }^{1,4}$

\section{* Correspondence:}

mpersinger@laurentian.ca

${ }^{1}$ Clinical Neuroscience Laboratory, Laurentian University, Sudbury, ON P3E 2C6, Canada

${ }^{2}$ Behavioural Neuroscience,

Laurentian University, Sudbury, ON

P3E 2C6, Canada

Full list of author information is

available at the end of the article

\section{Abstract \\ Background: Since 1980 we have assessed individuals who report electromagnetic sensitivity and "hearing voices" who do not display formal thought disorder but are clearly distressed by their experiences. Their intellectual levels and memory scores are average to above average with no neuropsychological impairment although right prefrontal deficits have been discernable. They exhibit experimentally verifiable sensitivity to physiologically-patterned magnetic fields at intensities they could encounter in their habitats.}

Presentation of Hypothesis: Considering the previous evidence that the parahippocampal region in the right hemisphere is sensitive to geomagnetic and experimentally generated weak, physiologically-patterned magnetic fields, these individual should display anomalous activity within the temporal lobes according to classic electroencephalographic measurements but with specific enhancements of current source density as measured by Low Resolution Electromagnetic Tomography (LORETA) in the right parahippocampal region.

Testing of Hypothesis: A middle-aged woman who displayed characteristics typical of this population exhibited persistent and conspicuous enhanced power in the low beta-range over the temporal lobes and specific changes in current source densities within the left inferior temporal gyrus and right parahippocampal region.

Implications of Hypothesis: This configuration could be helpful to differentiate this population. Given the increasing density and complexity of electromagnetic fields generated by commercial and private sources the prevalence of this subpopulation is expected to increase during the next decades.

Keywords: LORETA (Low Resolution Electromagnetic Tomography), Ambient Electromagnetic Sensitivity, "Schizophrenic" Presentations, Quantitative EEG, Right Parahippocampal Current Density Anomalies 


\section{Background}

Over the last 35 years we have assessed clinically approximately twenty adult men and women who have reported they "heard voices" and were sensitive to electromagnetic fields that the patients attributed to the cause of these voices. Although such symptoms are often considered classic Schneiderian indicators of formal thought disorders, none of the individuals we assessed met the criteria of the various DSM variants over the years. Most of these individuals were well educated (Bachelors, Masters or Doctoral Degrees), articulate, logical, and stable. Psychometric indicators, such as the MMPI (Multiphasic Minnesota Personality Inventory), indicated elevated scores for eccentric thinking (typical of creative writers) but the general profile was not sufficient for a psychiatric-level diagnosis.

Because a focus of our clinical laboratory (Persinger, 2001; 2003; St-Pierre and Persinger, 2006) is experimentally eliciting the "sense of a presence" (the feeling that a "Sentient Being" is proximal to the experient) by physiologically-patterned magnetic fields over the right temporal lobe at intensities that could be encountered in the modern habitat, we have been able to test the "electromagnetic sensitivity" directly. In most cases transcerebral applications of weak physiologically patterned magnetic fields generated from computer software across the temporal lobes while the patient sits blindfolded within a comfortable chair within a darkened quiet room (an acoustic chamber) encourage the feeling of "others" or the "sensed presence" in sensitive subjects as indicated by their electroencephalographic (EEG) responsiveness (Saroka and Persinger, 2013). Normal individuals with elevated complex partial epileptic-like indicators (Persinger and Makarec, 1993) are more responsive according to both strip chart electroencephalographic and subjective measures. The maximum strength of the magnetic fields within the space occupied by the brain is about 1 microTesla (10 milliGauss) which is within the range to which the brain is exposed near computers and a variety of modern electronic technologies including audio sources inserted over or within the outer ears.

For those patients who are actively experiencing, according to their verbal reports, inner voices application of these physiologically patterned fields with equal intensities across both temporal lobes markedly attenuates the numbers of "different voices" or eliminates their occurrence. Asymmetrical application with greater intensity over the right hemisphere enhances or initiates the experiences. The required duration of exposure is about $15 \mathrm{~min}$ to $30 \mathrm{~min}$. Cognitive interruption of the patient during this period markedly attenuates the effectiveness of the treatment. For both the induction of the sensed presence, the feeling of thought intrusion, and depersonalization ("out of body experiences") the optimal point duration of the serial voltages that produce the current through the solenoids (small reed switches) that generate the magnetic fields applied over the temporal lobes is $3 \mathrm{~ms}$ (see Saroka and Persinger, 2013 for details). This duration has been experimentally demonstrated to induce analgesic effects in rodents and is equivalent to about $4 \mathrm{mg}$ per kg of morphine (Martin et al. 2004). Recently Karbowski et al. (2012) found that applications of these types of fields to malignant cell cultures diminished their growth rates but did not affect non-malignant cell activity.

Within both psychiatric and non-psychiatric populations the report of a sensed presence has been associated with the transient occurrence of coherence between the right and left temporal lobes (Saroka and Persinger, 2013). Our metaphor for 
this effect is that the right hemispheric equivalence of the left hemispheric sense of self "intrudes" into or intercalates with awareness. Strip-chart and more recently quantitative electroencephalography (QEEG) measurements have supported this general hypothesis (Persinger and Tiller, 2008; Persinger et al., 2009). The availability of s_LORETA (Low Resolution Electromagnetic Tomography) has allowed the visualization of changes in source current density within the cerebral volume as a function of discrete $1 \mathrm{~Hz}$ frequency bands between 1 and $40 \mathrm{~Hz}$.

\section{Presentation of the Hypothesis}

Mulligan et al. (2010) while replicating the innovative measurements of Babayev and Allahverdiyeva (2007), showed that quantitative shifts in gamma and theta activity over the right (frontal) hemisphere were strongly correlated with both geomagnetic activity and ionospheric power density. The sensitivity of the right hemisphere was demonstrated during whole body exposure to experimentally-generated, weak 7 to $8 \mathrm{~Hz}$ magnetic fields (Mulligan and Persinger, 2012). The stratum stellare of Stephan (Layer II) of the entorhinal cortices of the human parahippocampal gyrus contains star-shaped cells that are organized into small elevations (verrucae gyri hippocampi) on the cortical surface that can be recognized visually (Gloor, 1997) . These cells exhibit intrinsic oscillations within the $8 \mathrm{~Hz}$ range with amplitudes whose energies approach that associated with the loss or gain (Lindauer Threshold) of 1 bit of information. This is the fundamental frequency for the Schumann Resonance (7-8 Hz with increasing harmonics every $\sim 6 \mathrm{~Hz}$, i.e., $13-15 \mathrm{~Hz}, 19-21 \mathrm{~Hz}, 25-27 \mathrm{~Hz}$ ) that is generated within the biosphere between the earth's surface and ionosphere.

Decades ago Koenig et al. (1981) emphasized the marked similarity between delta, alpha, and beta patterns in the human EEG and similar patterns generated within the natural environment. That resonance interactions could occur has been reported by Booth et al. (2005, 2008). Persinger and Saroka (2014), employing standardized LORETA inferences, measured marked enhancements of power within the $4 \mathrm{~Hz}$ to $10 \mathrm{~Hz}$ band within the right caudal hemisphere for only a narrow range of applied intensities. Although the experimental field strengths of a physiologically-patterned magnetic field applied across the temporal lobes ranged from $<1 \mathrm{nT}$ to $7,000 \mathrm{nT}$, only the 5 to $20 \mathrm{nT}$ increment produced these cerebral effects. Persinger and Saroka (2014) calculated that the likely energetic values represented within the cerebral volume indicated the potential role of Schumann-related frequencies.

Integration of these data in conjunction with that measured for special individuals who report frequent intrusive but benign experiences (Persinger and Saroka, 2012), suggested that this sub-population of people with this constellation of symptoms and experiences should display enhanced power within the higher harmonics (20 to $24 \mathrm{~Hz}$ range) of the Schumann band over the temporal lobes compared to other regions according to standard QEEG measures. This enhancement would be relatively continuous and reliably evident by repeated EEG measurements. In addition LORETA should demonstrate enhanced current density particularly within the alpha-beta transition (about $10 \mathrm{~Hz}$ to $15 \mathrm{~Hz}$ ) within the region of the right parahippocampus.

\section{Testing the Hypothesis}

A middle age female reported a history of multiple voices that were interpreted by her as "calls for help" from people trapped within a facility controlled by an intelligence 
system. The "voices" were also associated with "hearing-knowing" sequences of numbers that were organized in clusters typical of cell phone addresses. These number sequences, which she recorded, would be "received" for several minutes to an hour during any given episode. We randomly selected about 20 of these from hundreds of numbers and found most of them were or had been operational cell phone numbers (some were land lines).

She was formally tested over a three day period. Thorough neuropsychological assessment of this subject, like other subjects who presented with this profile, indicated an average level of intelligence and above average general memory and verbal fluency scores. Standardized scores from approximately 80 subtests (see Tiller et al. 2013) were within the average range. The only below normal scores were associated with tests by which right prefrontal function is inferred, such as the delayed (30 min) recall of the Rey-Ostereith Figure and the Conditioned Spatial Association Task. This cerebral region has been a major focus of our research because it is centrally involved with processing of the sense of "self" (Keenan et al., 2000). There was no formal Halstead-Reitan-type neuropsychological impairment. Her standardized scores for the MMPI were within the normal range.

During each QEEG recording the patient sat alone in a comfortable chair that was housed within a dimly lit acoustic chamber $\left(13 \mathrm{~m}^{3}\right)$. The computer displaying the brain activity was outside of the closed chamber. The patient wore a lapel microphone by which she interacted with the clinicians outside the chamber. All comments were tape-recorded. Brain electrical activity was monitored using a Mitsar 201 amplifier equipped with a 19-channel Electro-Cap International with $19 \mathrm{AgCl}$ sensors. For the type of analyses we pursue, which is primarily spectral density and microstates, we find this resolution to exhibit sufficient clinical information with such large effects. Measurements from 19 sites (Fp1, Fp2, F7, F3, Fz, F4, F8, T3, C3, Cz, C4, T4, T5, P3, Pz, P4, T6, O1, O2) consistent with the International Standard of Electrode Placement were obtained. Impedance for all measurements was maintained below 5 $\mathrm{kOhms}$. The data recorded from the amplifier was delivered to a Dell laptop (outside of the chamber) equipped with WinEEG v.2.8 that produced a digital copy of the recorded voltages. The sample rate was $250 \mathrm{~Hz}$.

Two basic analyses were performed on the data. One analysis was conducted in order to discern if regional differences in current source density occurred when referenced to an age-matched BRL database readily available within the sLORETA platform. For this analysis, 50x3-second segments of data were extracted and processed according to the specifications of recommended practices of the software. In brief this included 1) resampling the data to $100 \mathrm{~Hz}$ with 256 data points (approx. 2.56 seconds) 2) computing the average cross-spectra across all 50 samples, 3) computing current source density from this cross-spectral file and 4) comparing the results against the BRL normative database.

The second analysis was performed in order to discern if functional differences within the right, left or bilateral parahippocampal regions were apparent while the patient was relaxing with eyes closed, talking normally or "receiving transmissions" in light of the hypothesis and because of a connection between activity within the parahippocampus and naturally-occurring geomagnetic activity (Saroka and Persinger 2013, 2014) and its prominent role as an intermodal integrator of experience before the initiation of long-term memory processes within the hippocampus. For this analysis $8 \times 4$-second baseline segments as well as $8 x 4$-second 'receiving' segments were collected approximately 1-1.5 minutes before an "end of transmission" and were entered into sLORETA 
(Pasqual-Marquis, 2002) for computation of current source density $\left(\mu \mathrm{A} / \mathrm{mm}^{2}\right)$ within the right and left parahippocampal regions for the classical frequency bands used in more conventional electroencephalographic studies [i.e. delta $(1.5-4 \mathrm{~Hz})$; theta $(4-7 \mathrm{~Hz})$; low-alpha $(7-10 \mathrm{~Hz})$; high-alpha $(10-13 \mathrm{~Hz})$; beta-1 $(13-20 \mathrm{~Hz})$; beta-2 $(20-25 \mathrm{~Hz})$; beta-3 $(25-30 \mathrm{~Hz})$; gamma $(>30 \mathrm{~Hz})]$. The methods employed were identical to those reported by Saroka et al., 2013. These data were then entered into SPSS for statistical analysis.

The patient had some control over the occurrence of the intrusive voices, although at times they could occur spontaneously. When she "heard" these voices her verbal behaviour would become more monotonic ("machine-like") and her pronoun usage shifted from "I" to "we". QEEG indicated a persistent (measured on different days) 21-23 Hz higher amplitude $(\sim 70 \mu \mathrm{V})$ activity over T3 and T7 when she referred to "I" at which times she exhibited normal prosody.

When the "we state" was reported there was marked fast, high amplitude 17-23 Hz activity from both T3 and T4 as shown in Figure 1. In addition she reported "transmissions" which usually involved more complex information from these voices as well as series of numbers. When this occurred there was a "normalization" of the EEG (Figure 2). When the "transmissions ended", the unusual profile of T3 and T4 enhancements returned. There was additional similar activity over F7 and F8 which was transient. She was not talking during this period (after panel 1623).

However standardized scores for the current source densities for LORETA BRL comparison indicated below normal $(\mathrm{z}<-3.00$; based on software norms) delta "power" within the left inferior temporal gyrus, above normal $(\mathrm{z}>3.00)$ alpha power within the left inferior and middle frontal gyri, and marked abnormally elevated $(\mathrm{z}>4.00)$ power within the 17 to $23 \mathrm{~Hz}$ range within the right parahippocamapal and insular region. Figure 3 shows the results of the comparison between baseline and 'receiving' QEEG profiles using one-way analysis of variance and highlights the enhancement of $13-20 \mathrm{~Hz}$ activity within the right parahippocampal region. The F statistic indicates category differences. The omega-squared estimate $(94 \%)$ refers to the amount of variance explained in the change of current source density between intervals when she was in the "I state" vs the "we state" when "information" was being received according to her reports.

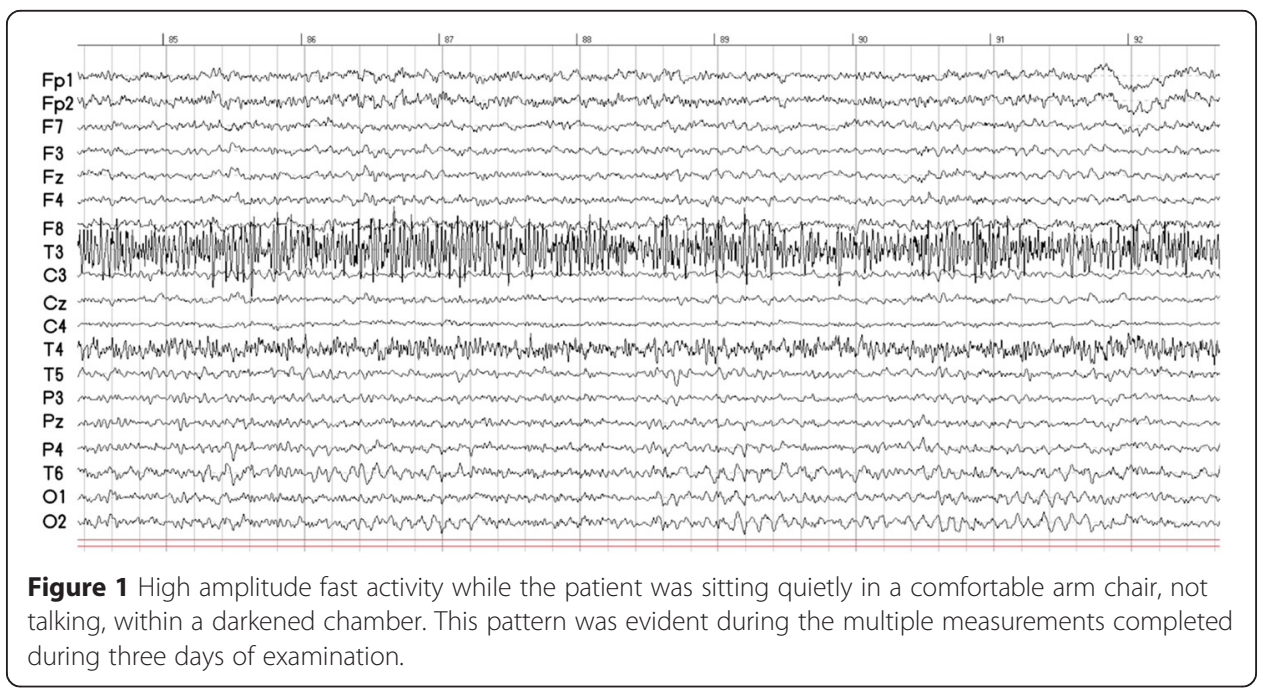




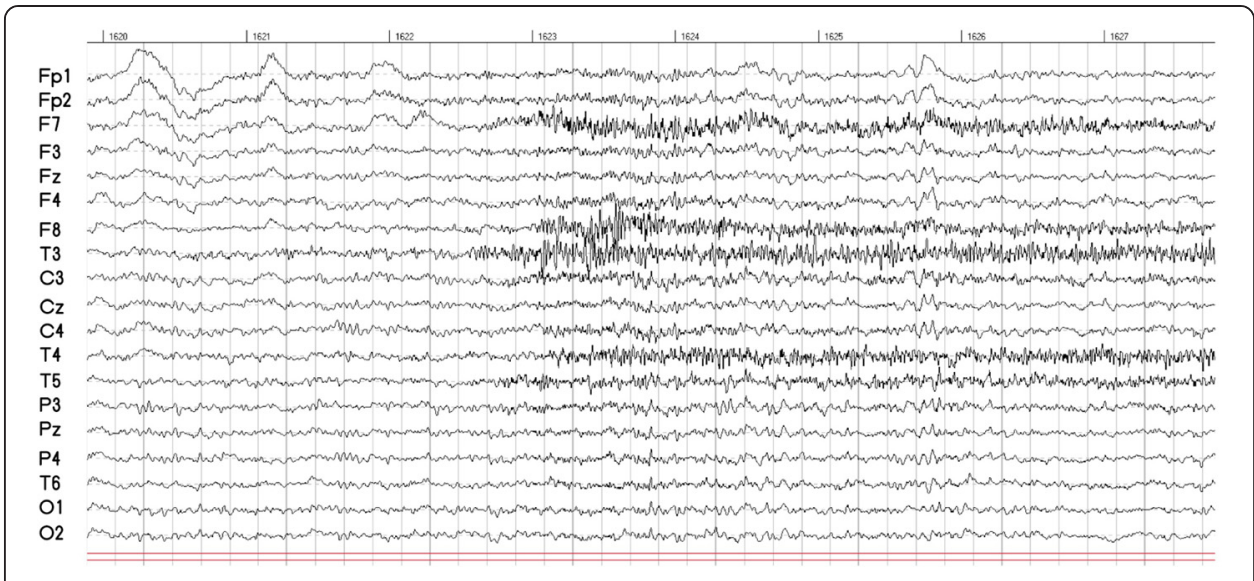

Figure 2 During some periods when she was reporting multiple "voices" and series of numbers that patient described as "transmissions", the EEG normalized. When the "transmission terminated" (panel 1623) to employ her phraseology, there was the return of the T3 and T4 activity with additional frontal contributions.

During the experiences that would be classically labeled as "intrusions" the activation score for the low beta power within the right parahippocampal region more than doubled. This area and related hemispheric discrepancy are similar to that associated with "panics" that can occur suddenly in this group of patients.

One of the positive reports by the patient was that simply sitting in the closed acoustic chamber (which is also a Faraday room) attenuated the loudness of the annoying "ringing" that she has experienced for years. On several occasions, while sitting quietly in the chamber for "baseline" measurements and while wearing the apparatus by which we have experimentally induced the "sense of presence" in most normal volunteers over the last two decades (Persinger, 2003; Saroka and Persinger, 2013; St-Pierre and Persinger, 2006), the frequency-modulated magnetic fields (Booth et al., 2005) were activated over the temporoparietal regions without the subject's knowledge. We selected two patterns, one with an accelerating frequency modulation ("burst- $x$ ") and one with a decelerating frequency modulation ("Thomas pulse") which are the major components of our protocols for eliciting the sensed presence. When she was exposed to the latter temporal pattern she experienced an almost immediate oppression in her chest and a headache. When the burst-x fields were applied she felt "fine" within about $10 \mathrm{~s}$. During the presence of the fields the spontaneous narratives referring to the "we state" stopped. The prosody of her voice was typical of normal speech.

\section{Implications of the Hypothesis}

These cerebral patterns have been so consistent, at least in the population that we have assessed over the years with increasingly more quantitative technology, that they might also be apparent to other clinicians who have access to and expertise with QEEG and LORETA. Our interpretation is that the lowered base power within the delta range (upon which higher frequencies can be strongly dependent) within the left temporal lobe facilitates the conditions for inter-temporal lobe coherence and the experiences of "others" (Booth and Persinger, 2009). The enhancement of power within the left inferior frontal region, traditionally associated with expression of overt language (Petkov et al., 2009), could 


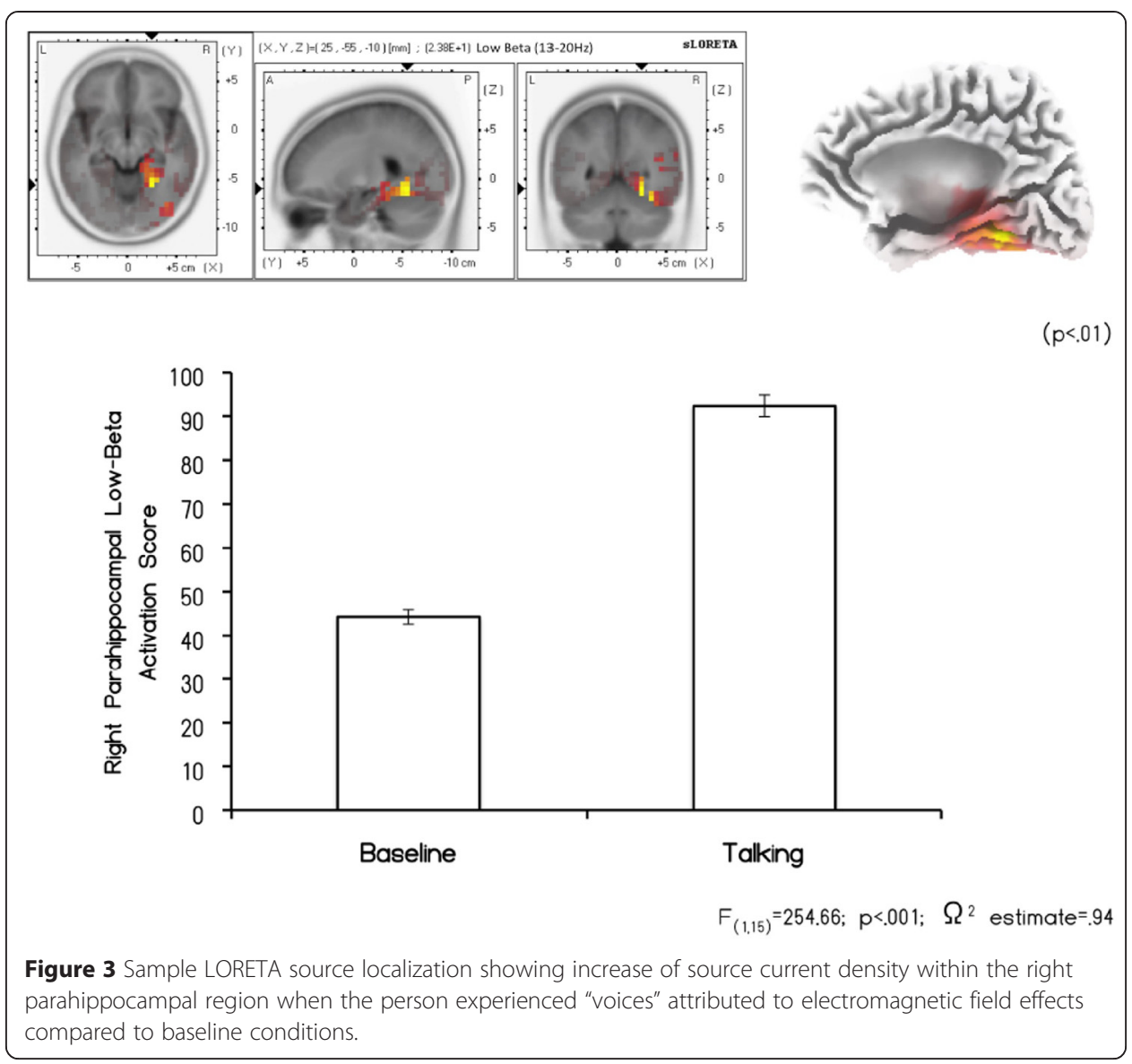

encourage the amplification of the person's own array of "articulemes". They are the neurocognitive patterns accompanying discrete neural activity that initiate sequences of stylopharyngeal and laryngeal muscle contractions. Anomalous organizations within the right prefrontal (Keenan et al., 2000) could increase the probability that the reconstruction of experiences would be attributed to non-self sources.

However what is very different about these individuals is their sensitivity to ambient electromagnetic fields. More than fifty years ago Frey (1962) demonstrated that GHz range transmitted fields pulsed within the EEG-frequency band could induce auditory experiences. We have measured $\mathrm{GHz}$ power densities in the $10 \mathrm{~mW} \cdot \mathrm{m}^{-2}$ range frequently within modern habitats. Across the width of neuronal soma the energy would be about 1 picoJoule per second which is the magnitude of energy provided per second by glucose metabolism. The energies from a single action potential or from the force between the potassium ions that contribute to the resting membrane potential are almost a billion times smaller (Persinger, 2010). With the increasing myriad of communication transmissions novel "beats" (the subtraction of two frequencies) will emerge that are within the extremely low frequency range that could directly resonate (at least transiently) with the electrophysiology of the human brain.

Both the magnetic field intensity and potential differences generated by the human brain and by the Schumann Resonance (Nickolaenko and Hayakawa, 2014) are within the same order of magnitude. Although the amplitudes are considered by some researchers to be "too small" to be biologically relevant, Saroka and Persinger (2014) have shown 
real-time coherence between QEEG measurements and Schumann power densities can be measured directly. These coherences across the Schumann harmonics, such as the ones displayed by the patient, occur for about $300 \mathrm{~ms}$ once every approximately $30 \mathrm{~s}$, the typical decay time for short-term memory processes (Duff and Brown-Schmidt, 2012). The LORETA profiles and the most recent technology suggest that patients who present with "hearing voices"' attributed to electromagnetic fields but without formal thought disorder or neuropsychological deficits should be assessed by this quantitative method. We predict they should show the profile described here and would demonstrate fluctuations in their reports and symptoms when either the appropriate, weak physiologically-patterned magnetic fields are applied across the temporal lobes or specific intensities of geomagnetic activity occur. Recently the third author recorded a significant "displacement" (decreased by about $2 \mathrm{~Hz}$ ) in the third harmonic (19-21 Hz) during a particularly energetic $(K>6)$ geomagnetic storm.

We predict an increase in prevalence of this population whose incidence will likely occur as sporadic spatial-temporal clusters that reflect critical environmental conditions. We cannot discern at present whether the increased prevalence will be caused by the increased electromagnetic complexity within the $21^{\text {st }}$ century environment (Whissell and Persinger, 2007) or if the environment is emerging that is congruent with the sensitivity of a more or less fixed proportion of the population that has always been present. There are no a priori means at this time that would allow discernment of the validity of the patient's experiences or their origin. However if the initial neuroelectrical disruptions are correlated with direct changes from the artificial electromagnetic fields within the environment, sporadic geomagnetic activity or enhancements of Schumann parameters, the more optimal treatment would involve lower dosages of antiepileptic compounds rather than traditional antipsychotic pharmacology (Hines, et al., 1995; Roberts and Franzen 2001; Persinger, 2000). In our clinical experience the latter can exacerbate the symptoms in this particular population.

Competing interests

There are no competing interests by any of the authors.

Authors' contributions

All authors contributed equally to the content of this manuscript. All author read and approved the final manuscript.

Acknowledgements

We thank the many patients, participants, and volunteers who had the courage to report their experiences and to pursue, vigorously, realistic solutions for their dilemmas. The technical contributions over the years of Dr. Kate Makarec, Sandra G. Tiller, Professor Stanley Koren, and Dr. Blake T. Dotta are acknowledged.

\footnotetext{
Author details

${ }^{1}$ Clinical Neuroscience Laboratory, Laurentian University, Sudbury, ON P3E 2C6, Canada. ${ }^{2}$ Behavioural Neuroscience, Laurentian University, Sudbury, ON P3E 2C6, Canada. ${ }^{3}$ Biomolecular Sciences, Laurentian University, Sudbury, ON P3E 2C6, Canada. ${ }^{4}$ Interdisciplinary Human Studies Programs, Laurentian University, Sudbury, ON P3E 2C6, Canada.

Received: 4 February 2015 Accepted: 30 March 2015

Published online: 01 July 2015

References

Babayev E, Allahverdiyeva A (2007) Effects of geomagnetic activity variations on the physiological and psychological state of functionally healthy humans: some results of Azerbaijani studies. Adv Space Res 40:1941-1951

Booth J, Persinger M (2009) Discrete shifts within theta band between the frontal and parietal regions of the right hemisphere and the experiences of a sensed presence. J. Neuropsychiatry Clin Neurosci 21:279-283

Booth J, Koren S, Persinger M (2005) Increased feelings of sensed presence and increased geomagnetic activity at the time of the experience during exposures to transcerebral weak complex magnetic fields

Booth J, Koren S, Persinger M (2008) Increased theta activity in quantitative electroencephalographic (QEEG) measurements during exposure to complex weak magnetic fields. Electromag Biol Med 27:426-436
} 
Duff C, Brown-Schmidt S (2012) The hippocampus and the flexible use and processing of language. Front Human Neurosci 6: article 69

Frey A (1962) Human auditory systems response to modulated electromagnetic energy. J App Physiol 17:689-692

Gloor P (1997) The temporal lobe and the limbic system. London, Oxford

Hines M, Kubu C, Roberts R, Varney N (1995) Characteristics and mechanism of epilepsy spectrum disorder: an explanatory model. Appl Neuropsychol 2:1-6

Karbowski L, Harribance S, Buckner C, Mulligan B, Koren S, Lafrenie PM (2012) Digitized quantitative electroencephalographic patterns applied as magnetic fields inhibit melanoma cell proliferation in culture. Neurosci Lett 523:131-134

Keenan J, Wheeler M, Gallup G, Pascual-Leone A (2000) Self-recognition and the right prefrontal cortex. Trends Cog Sci 4:338-334

Koenig H, Krueger A, Lang S, Sonning W (1981) Biologic effects of environmental electromagnetism. Springer, N.Y.

Martin J, Koren S, Persinger M (2004) Thermal analgesic effects from weak, complex magnetic fields and pharmacological interactions. Pharm Biochem Behav 78:217-227

Mulligan B, Persinger M (2012) Experimental simulation of the effects of sudden increases in geomagnetic activity upon quantitative measures of brain activity: validation of correlational studies. Neurosci Lett 513:151-154

Mulligan B, Hunter M, Persinger M (2010) Effects of geomagnetic activity and atmospheric power variations on quantitative measures of brain activity: replication of the Azerbaijani studies. Adv Space Res 45:940-948

Nickolaenko A, Hayakawa M (2014) Schumann resonance for Tyros. Springer, Heidelberg

Pasqual-Marquis R (2002) Standardized low resolution brain electromagnetic tomography (sLORETA): technical details, methods and findings. Exp Pharmacol 34:D5-D12

Persinger M (2000) Subjective improvement following treatment with carbamazeine (Tegretrol) for a subpopulation of patients with traumatic brain injuries. Percep Mot Skil 90:37-40

Persinger M (2001) The neuropsychiatry of paranormal experiences. J Neuropsychiat Clin Neurosci 13:515-524

Persinger M (2003) The sensed presence within experimental settings: implications for the male and female concept of self. J Psychol 137:5-16

Persinger M (2010) $10^{-20} \mathrm{~J}$ as a neurmolecular quantum in medicinal chemistry: an alternative approach to myriad molecular pathways. Cur Med Chem 17:3094-3098

Persinger M, Makarec K (1993) Complex partial epileptic-like signs as a continuum from normals to epileptics: normative data and clinical populations. J Clin Psych 49:33-45

Persinger M, Saroka K (2012) Protracted parahippocampal activity associated with Sean Harribance. Int J Yoga 5:140-145

Persinger M, Saroka K (2014) Quantitative support for convergence of intrinsic frequencies from applied magnetic fields and "noise" fluctuations of Newton's gravitational value within the human brain. Int Lett Chem Phys Astron 19:181-190

Persinger M, Tiller S (2008) A prototypical spontaneous "sensed presence" of a Sentient Being and concomitant electroencephalographic activity in the clinical laboratory. Neurocase 14:425-430

Persinger M, Hoang V, Baker-Price L (2009) Entrainment of Stage 2 sleep spindles by weak transcererbal magnetic stimulation of an "epileptic" woman. Electromag Biol Med 28:374-382

Petkov C, Logothetis N, Obleser J (2009) Where are the human speech and voice regions, and do other animals have anything like them? Neuroscientist 15:419-429

Roberts R, Franzen VN (2001) Theta bursts, closed head injury, and partial seizure-like symptoms. A retrospective study. App Neuropsychol 8:140-147

Saroka K, Persinger M (2013) Potential production of Hughlings Jackson's "parasitic consciousness" by physiologically-patterned weak transcerebral magnetic fields: QEEG and source localization. Epilep Behav 28:395-407

Saroka K, Persinger M (2014) Quantitative evidence for direct effects between earth-ionosphere Schumann resonances and human cerebral cortical activity. Int Lett Chem Phys Astron 20(2):166-194

Saroka K, Caswell J, Lapointe A, Persinger M (2013) Greater encephalographic coherence between the left and right temporal lobe structures during increased geomagnetic activity. Neurosci Lett 7:126-130

St-Pierre L, Persinger M (2006) Experimental facilitation of the sensed presence is predicted by specific patterns of applied magnetic fields not suggestibility: re-analysis of 19 experiments. Int J Neurosci 16:1-18

Tiller S, St-Pierre L, Persinger M (2013) Absence of quantitative improvement in neuropsychological profiles in patients who exhibit moderate brain impairment: comparisons of cross-sectional and longitudinal data (1 through 4 years post injury). J Behav Brain Sci 3:225-238

Whissell P, Persinger M (2007) Emerging synergisms between drugs and physiologically-patterned weak magnetic fields: implications for neuropharmacology and the human population in the twenty-first century. Cur Neuropharm $5: 278-288$ 